\title{
Appraisal of Awareness and Attitude of School Teachers towards Emergency Management of Dental Trauma and Tooth Avulsion Replantation
}

\author{
Shivani Kharpate ${ }^{1}$, Nilesh Rathi², Pooja Vilas Gomase ${ }^{3}$, Sudhindra Baliga ${ }^{4}$, Nilima Thosar ${ }^{5}$ \\ ${ }^{1}$ Sharad Pawar Dental College, Datta Meghe Institute of Medical Sciences, Wardha, Maharashtra, India. \\ ${ }^{2}$ Department of Pedodontics, Sharad Pawar Dental College, Datta Meghe Institute of Medical Sciences, \\ Wardha, Maharashtra, India. ${ }^{3}$ Sharad Pawar Dental College. Datta Meghe Institute of Medical Sciences, \\ Wardha, Maharashtra, India. ${ }^{4}$ Department of Pedodontics, Sharad Pawar Dental College, Datta Meghe \\ Institute of Medical Sciences, Wardha, Maharashtra, India. ${ }^{5}$ Department of Pedodontics, Sharad Pawar \\ Dental College, Datta Meghe Institute of Medical Sciences, Wardha, Maharashtra, India.
}

\section{ABSTRACT}

\section{BACKGROUND}

Health education is an essential aspect for prevention of oral health problems in school children. Dental trauma can result in damaging the tooth, periodontal ligaments, and sometimes even alveolar bone and gingiva. These traumatic injuries to the anterior teeth in young children are tragic but are an ignored problem. They can result in aesthetic and functional problem with possible impact on child's quality of life as anterior teeth are essential for phonetics, mastication, integrity of supporting tissue and also mental and psychological wellbeing of children. This dental emergency can be best handled by the school teachers and the staff members when it happens in a school. So, the study was conducted to assess the knowledge and attitude of the school teachers regarding the emergency management of dental trauma and sensitize them on the subject.

\section{METHODS}

This is a pre- and post-test study performed using self - designed questionnaire provided to the school-teachers of Wardha district.

\section{RESULTS}

Knowledge of $98 \%$ teachers increased after the activity whereas knowledge of $2 \%$ of school teachers was the same as earlier.

\section{CONCLUSIONS}

Sensitization of the school teachers improved their awareness and attitude to manage the dental trauma and avulsion injury of teeth in school.

\section{KEY WORDS}

Tooth Avulsion, Emergency Dental Traumatic Care, School Teachers
Corresponding Author: Dr. Shivani Kharpate, Plot no. 7, Rukhmini Nagar, Sindhi Meghe, Wardha - 442001, Maharashtra, India.

E-mail: shivanikharpate2@gmail.com

DOI: $10.14260 /$ jemds/2020/647

How to Cite This Article:

Kharpate S, Rathi N, Gomase PV. et al Appraisal of awareness and attitude of school teachers towards emergency management of dental trauma and tooth avulsion replantation.J Evolution Med Dent Sci 2020;9(40):2951-2954, DOI: 10.14260/jemds/2020/647

Submission 10-05-2020,

Peer Review 13-08-2020,

Acceptance 20-08-2020,

Published 05-10-2020.

Copyright (C) 2020 Shivani Kharpate et al. This is an open access article distributed under Creative Commons Attribution License [Attribution 4.0 International (CC BY 4.0)] 


\section{BACKGROUND}

Health education is an essential aspect for prevention of oral health problem in school children. ${ }^{1}$ Dental trauma can result in damaging the tooth, periodontal ligaments and sometimes even alveolar bone and gingiva. These traumatic injuries to the anterior teeth in young children are tragic but are ignored problem. This traumatic injuries cause increase in mortality of teeth. During the age 8 - 15 children are actively included in outdoor activities. During the school periods children often play outdoor games. Though this activities are marked to be profitable in their growth and development, they increase the possibilities of injuries. This results into aesthetic and functional problem with possible impact on child's quality of life as anterior teeth are essential for phonetics, mastication, integrity of supporting tissue and also mental and psychological wellbeing of children. Males generally report more traumatic Dental Injuries than females because of their active participation in sports and games. ${ }^{2}$ Teeth most commonly involved are Maxillary central incisor - $37 \%$, Mandibular central incisors -18 \%, Mandibular lateral incisors - $6 \%$, Maxillary lateral incisors - $3 \%$. The pre - disposing factors like increased overjet, protrusion, material deprivation, human behaviour like risk taking and hyperactivity disorder. Traumatic Dental Injuries involving hard and soft tissue require emergency treatment. Actions ensuring pain control, restoration of aesthetic and function and prevention of psychological consequences are required. The dental emergency can be best handled by the school teachers and the staff members. So, the study was conducted to assess the knowledge and attitude of the school teachers regarding the emergency management of dental trauma in school. ${ }^{3}$ Recently Aloe vera gel is used as a storage medium in maintaining viability of periodontal ligament cells. ${ }^{4}$ Apart from school teachers Anganwadi worker due to their close contact with children and women also should have knowledge to manage emergency dental trauma. ${ }^{5}$

\section{METHODS}

This was an interventional study done in the Department of Paediatric and Preventive Dentistry, Sharad Pawar Dental College, Sawangi (Meghe), Wardha, India. After collecting the list of all schools in Wardha district of Maharashtra two school were randomly selected as study area. Study materials - Selfadministered questionnaire. pen and notebook.

After obtaining the permission from principal of the school for conduction of the study. Fifty qualified teachers of primary and secondary schools present during the visit were explained regarding the study and the prepared questionnaire was provided to them. Purpose and methodology of study was explained to the teachers. This questionnaire in print was provided to the teachers in the staff room during the school hours to assess the knowledge of teachers regarding dental traumatic care and tooth avulsion replantation. The questionnaire (pre - test) was collected and analysed and the teachers were educated in brief about the emergency management of dental trauma and tooth avulsion replantation followed by discussion of the questionnaire. Then after a period of one month another questionnaire (post - test) was provided to the school teachers. After collecting post - test, pre - test and post - test were compared which gave pre sensitization and post-sensitization score and the knowledge regarding the topic was analysed using frequency distribution.

\section{Statistical Analysis}

As the study was based on the knowledge of the school teachers regarding tooth avulsion and dental trauma the variables of this study were 1 . Increase in the knowledge of the school teachers after the interaction or 2. No increase in the knowledge. We assume the knowledge of all the teachers will be increased after the interaction session as the objective was to check the improvement in knowledge of teachers regarding the topic Frequency distribution of descriptive statics was used to compare pre and post test result. The values showed in the table and graphs provided depicts the percentage of correct answer. Through frequency distribution it was concluded that the knowledge of $98 \%$ of teachers was increased and knowledge of $2 \%$ teachers was not increased. As shown in Fig. 1

\section{RESULTS}

\begin{tabular}{|lcc|}
\hline Que 1. Do you know about tooth Avulsion or & Yes & No \\
Knocked out Tooth? & $18(36 \%)$ & $32(64 \%)$ \\
Pre Test & $46(92 \%)$ & $4(8 \%)$ \\
Post Test & Yes & No \\
Que 2. Have you ever been provided information & $13(26 \%)$ & $37(74 \%)$ \\
regarding emergency Dental Traumatic Care? & $47(94 \%)$ & $3(6 \%)$ \\
Pre Test & Yes & No \\
Post Test & $34(68.0 \%)$ & $16(32.0 \%)$ \\
Que 3. Do you know that a knocked out tooth & $50(100 \%)$ & $0(0 \%)$ \\
can be replaced into the Socket?' & Pre Test & \multicolumn{2}{|c}{} \\
Post Test & \multicolumn{2}{|c|}{ Table 1. depicts knowledge of teachers regarding tooth avulsion } \\
\hline
\end{tabular}

In the pre-test $1^{\text {st }}$ question asked was 'do you know about tooth avulsion or knocked out tooth?' to which 18 (36\%) teachers answered YES and 32 (64\%) teachers answered NO. After the orientation in post - test the same question was repeated this time 46 (92\%) of teachers answered YES and 4 ( $8 \%$ ) teachers answered NO. The $2^{\text {nd }}$ question was 'Have you ever been provided information regarding emergency dental traumatic care? 13 (26\%) teachers answered YES and 37 (74 $\%)$ answered NO. In the post - test 47 (94\%) teachers were provided with information $3(6 \%)$ were not.

The $3^{\text {rd }}$ question was 'Do you know that a knocked out tooth can be replaced into the Socket?' 34 (68 \%) answered that it is possible and 16 (32\%) answered it is not possible. After the orientation when it was asked again then 48 (96\%) answered that it is possible whereas $2(4 \%)$ answered it as not possible.

The $4^{\text {th }}$ question was 'How immediately the tooth replantation should be done after the tooth comes out of the socket?' to which 1 (2\%) of teachers answered $5 \mathrm{~min}, 3(6 \%)$ of teachers answered $30 \mathrm{~min}, 3(6 \%)$ teachers answered 60 min, 7 (14\%) Teachers answered 24 hours, whereas $36(72$ $\%)$ of teachers did not know. When teachers were asked the same question after orientation in post - test 17 (34\%) teachers answered $5 \mathrm{~min}, 2$ (4\%) answered $30 \mathrm{~min}$., 29 (58 $\%$ ) answered $60 \mathrm{~min}, 0(0 \%)$ answered 24 hours whereas 2 (4 $\%)$ still did not know. As shown in Fig. 2. 

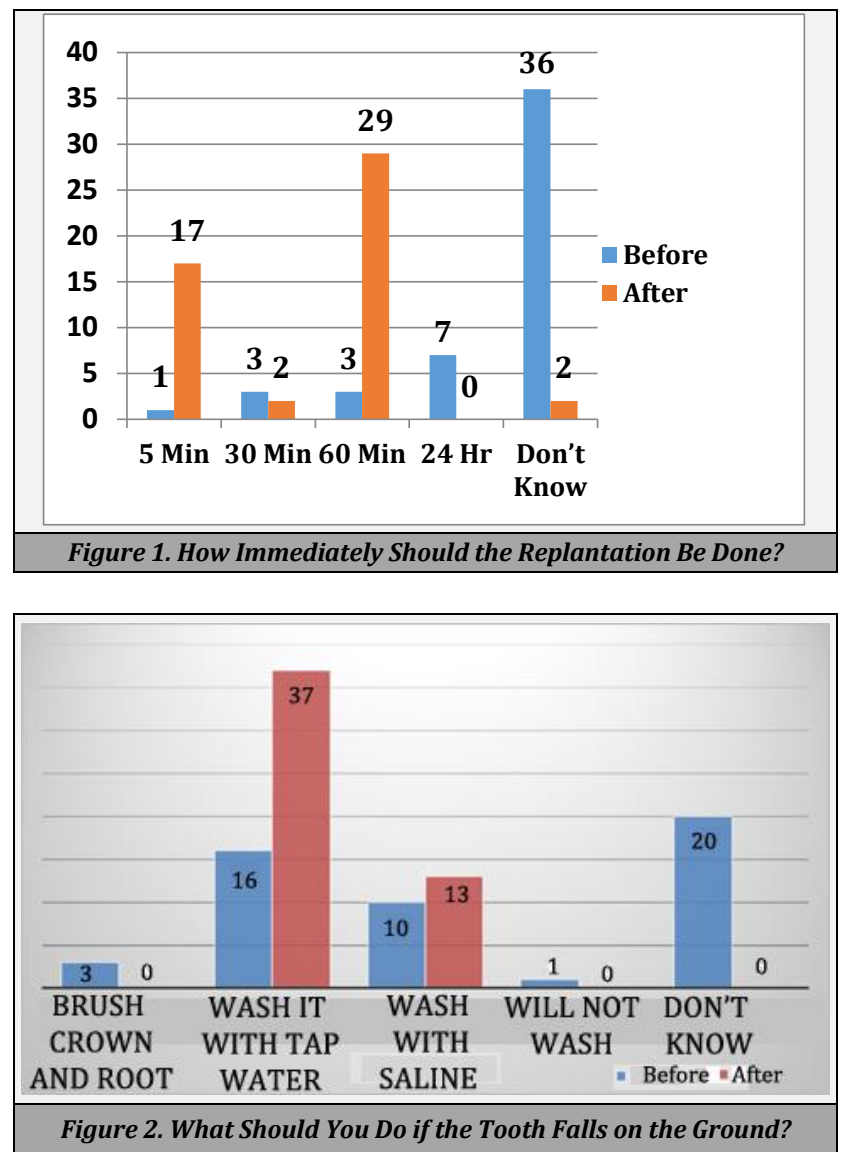

The $5^{\text {th }}$ question was 'What should you do if the tooth falls on the ground and get Dirty?' to which $3(6 \%)$ of teachers chose to brush crown and root, 17 (43\%) chose to wash it with tap water, 10 (20\%) chose to wash with saline, 1 (2\%) will not wash whereas 19 (38\%) did not know. When the same question was asked after the orientation in post - test $0(0 \%)$ chose to brush crown and root, 37 (64\%) would wash with tap water, $13(26 \%)$ would wash with saline, $0(0 \%)$ would not wash, 0 (0\%) did not know. As shown in Fig. 3.

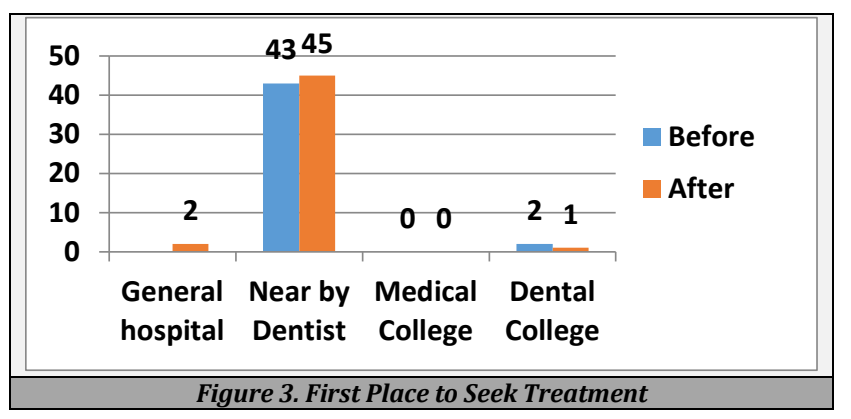

The $6^{\text {th }}$ question was 'First place to seek treatment?' for which 0 (0\%) chose General hospital, 43 (86\%) chose dear by dentist, $0(0 \%)$ chose medical college, $2(4 \%)$ chose dental college, $5(10 \%)$ chose emergency hospital. In post - test 2 (4 $\%)$ chose General hospital, 45 (90\%) chose Dentist nearby, 0 (0\%) chose Medical college, 1 (2\%) chose Dental college whereas $2(4 \%)$ chose emergency hospital. As shown in Fig. 4.

'Which transport medium will you use?' was the $7^{\text {th }}$ question for which, $5(10 \%)$ chose wet napkin, 20 (40\%) chose Cotton rolls, 6 (12\%) chose saline water, 2 (4\%) chose milk whereas 17 (34\%) did not know. After orientation in post -test $0(0 \%)$ chose wet napkin, 1 (2\%) chose cotton rolls, 9
(18\%) chose saline water, 40 (80 \%) chose milk and $0(0 \%)$ did not know. As shown in Fig. 5

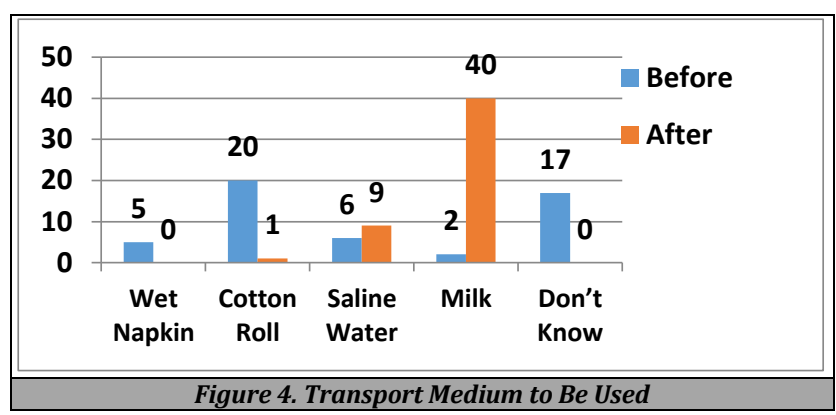

\begin{tabular}{|ccc|}
\hline Que 8. 'Which Tooth can be & Primary Tooth & Permanent Tooth \\
Replanted into the Socket?' & $4(8.0 \%)$ & $46(92.0 \%)$ \\
Pre Test & $1(2 \%)$ & $49(98 \%)$ \\
Post Test & Table 2. Which Tooth Can Be Replaced in the Socket? \\
\hline
\end{tabular}

The $8^{\text {th }}$ question was 'Which tooth can be replanted into the socket?' for which 4 (8\%) chose primary teeth and $46(92$ $\%)$ chose permanent teeth. After orientation in post - test the answer chose were such as $1(2 \%)$ teachers chose primary tooth, 49 (98\%) teachers chose permanent tooth. As shown in Table 2. In the post - test 9th question was "Was this orientation helpful in adding your knowledge regarding dental trauma?' to which 49 (98\%) answered yes whereas 1 (2\%) answered no.

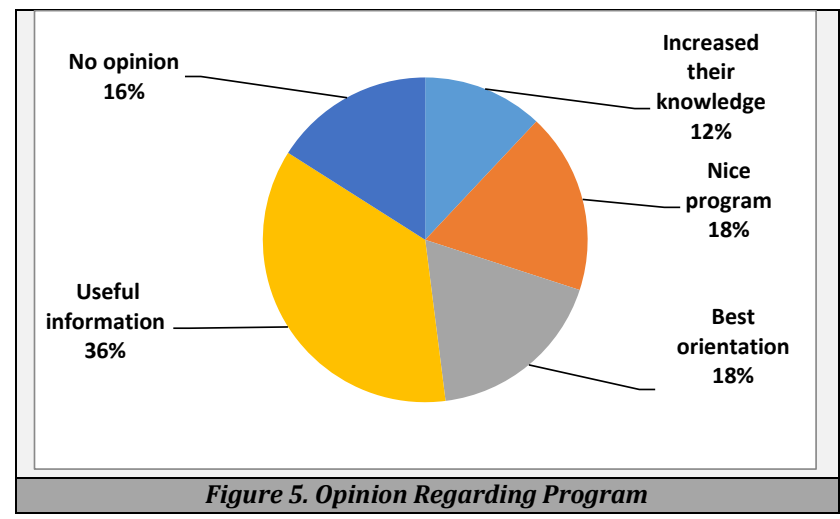

The last question was there in post-test asking about review and opinion of the teachers regarding this orientation, as the question was open ended it was categorized into 5 options 6 (12\%) said it increased their knowledge, 9 (18\%) said it was nice program, $9(18 \%)$ said it was best orientation, $18(36 \%)$ said it was use - full information and $8(16 \%)$ had no opinion.

\section{DISCUSSION}

The result of this study showed that the knowledge of the school teachers regarding tooth avulsion replantation increased after the orientation. Before the orientation only few school teachers knew about knocked out tooth but were not sure about their knowledge regarding this topic (Ref. Table 1). The PTI (Physical Training Instructor) of both the schools were made well aware regarding this topic earlier, they knew 
the duration, medium to be used, which tooth can be replanted, etc.

Else than the PTI of the school not much of the teachers knew about such treatment that is tooth avulsion replantation existed. Many teachers were surprised after listening to the topic. As they were never made aware of such facts. Also some of the teachers shared their experiences regarding such dental trauma to their loved - ones. Success of tooth avulsion replantation depends on minimal fracture and damage to the cells. In this study, in pre - test $36 \%$ of teachers knew that avulsed tooth can be place back into the socket whereas in post - test this number increased up to $92 \%$ in the post test (Ref. Table 1). $74 \%$ of the teacher were never provided with information regarding dental traumatic care as per result of pre - test. (Ref. Table 2) $68 \%$ of teachers knew that an avulsed tooth can be replanted inside the socket in pre - test, in post test $96 \%$ of teachers knew. (Ref. Table 1)

For the replantation of an avulsed tooth to be successful it is important to maintain its vitality. Replantation should be done as soon as possible with least damage to the cells. So as to know teachers knowledge regarding these topic in pre-test question 4 it was asked that how immediately the tooth replantation should be done after tooth comes out of socket to which $1(2 \%)$ of teachers answered $5 \mathrm{~min}, 3$ (6\%) of teachers answered $30 \mathrm{~min}, 3$ (6\%) teachers answered $60 \mathrm{~min}, 7$ (14\%) teachers answered 24 hours, where, as 36 (72\%) of teachers did not know. When teachers were asked the same question after orientation in post - test $17(34 \%)$ teachers answered 5 min, 2 (4\%) answered $30 \mathrm{~min}, 29$ (58 \%) answered $60 \mathrm{~min}, 0$ (0\%) answered 24 hours whereas 2 (4\%) still did not know (Ref. Fig. 2).

In most of the tooth avulsed cases reported the tooth falls on the ground and get dirty. It is important to know how to clean an avulsed tooth, when teachers were asked $63 \%$ teachers said that they will wash it with saline or water where as 38\% did not know in post - test $100 \%$ teachers chose to wash the tooth with tap water or saline (Ref. Fig. 3)

First place to seek treatment of $86 \%$ teachers was Dentist nearby, $10 \%$ chose emergency hospital. (Ref. Fig. 4)

Another important factor is transport medium to be used that can keep the tooth vital and should be easily available. 40 $\%$ of teachers preferred cotton rolls and $37 \%$ did not know.
After the orientation was done $18 \%$ preferred saline water where as $80 \%$ chose milk due to its availability (Ref. Fig. 5).

Regarding which tooth can be replanted $92 \%$ chose permanent tooth in pre - test by saying that primary tooth falls anyway (Ref. Table 8). In post - test $98 \%$ chose permanent tooth Teachers knowledge regarding the management of knocked out tooth was insufficient as pre - test (Ref. Table 2).

\section{CONCLUSIONS}

Knowledge of school teachers regarding the management of emergency dental trauma was insufficient due to which they were not capable of properly managing the knocked out tooth and child. During the orientation programme, most of the teachers carefully listened regarding the topic and were interested in increasing their knowledge. In post - test $98 \%$ teachers answered that their knowledge had improved.

Financial or Other Competing Interests: None.

\section{REFERENCES}

[1] Deolia S, Sen S, Ingole P, et al. Effect of conventional v/s game- based and app. Based oral health education on children's oral health - related knowledge. J Dent Res 2017;1(2):78-81.

[2] Gala V, Yadav T, Fernandes G. Knowledge and attitude of school teachers towards the management of a knocked out tooth. IOSR J Dent Med Sci 2018;17(6):39-43.

[3] Prasanna S, Giriraju A, Narayan NL. Knowledge and attitude of primary school teachers toward tooth avulsion and dental first aid in Davangere city: a cross- sectional survey. Int J Clin Pediatr Dent 2011;4(3):203-6.

[4] Fulzele P, Baliga S, Thosar N, et al. Evaluation of aloevera gel as a storage medium in maintaining the viability of periodontal ligament cells- an in vitro study. J Clin Pediatr Dent 2016;40(1):49-50.

[5] Gotarkar S, Ingole A. Knowledge of anganwadi worker with respect to early childhood development. J Datta Meghe Institute of Med Sci University 2018;13(4):168-70. 